\title{
Sampling Potential Energy Surfaces in the Condensed Phase with Many-Body Electronic Structure Methods
}

\author{
Rybkin, Vladimir V
}

\begin{abstract}
Sampling potential energy surfaces (PES) is pivotal for understanding chemical structure, energetics and reactivity and is of special importance for complex condensed-phase systems. Until recently such simulations based on electronic structure theory have been performed only by density functional theory and semiempirical methods. Many-body electronic structure methods, almost routinely used for molecules, have been practically unavailable for sampling PES in the condensed-phase. This has changed during the last few years, as efficient algorithms and software implementations for the evaluation of electronic energies and forces on atoms have been developed, allowing for geometry optimization, molecular dynamics and Monte-Carlo simulations, which was previously unthinkable. Herein, we introduce the theory and software developments and overview the applications in the field, the most encouraging results being obtained for aqueous chemistry. Requiring state-of-the-art computer resources PES sampling with many-body electronic structure methods in the condensed phase provides high-quality benchmarks and will gradually become more available due to fast progress in reduced scaling algorithms and computational technologies.
\end{abstract}

DOI: https://doi.org/10.1002/chem.201904012

Posted at the Zurich Open Repository and Archive, University of Zurich ZORA URL: https://doi.org/10.5167/uzh-183017

Journal Article

Accepted Version

Originally published at:

Rybkin, Vladimir V (2020). Sampling Potential Energy Surfaces in the Condensed Phase with Many-Body Electronic Structure Methods. Chemistry - A European Journal, 26(2):362-368.

DOI: https://doi.org/10.1002/chem.201904012 


\title{
SAMPLING POTENTIAL ENERGY SURFACES IN THE CONDENSED PHASE WITH MANY-BODY ELECTRONIC STRUCTURE METHODS
}

\author{
VLADIMIR V. RYBKIN
}

\begin{abstract}
Sampling potential energy surfaces (PES) is pivotal for understanding chemical structure, energetics and reactivity and is of special importance for complex condensed-phase systems. Until recently such simulations based on electronic structure theory have been performed only by density functional theory and semiempirical methods. Many-body electronic structure methods, almost routinely used for molecules, have been practically unavailable for sampling PES in the condensed-phase. This has changed during the last few years, as efficient algorithms and software implementations for evaluation of electronic energies and forces on atoms have been developed, allowing for geometry optimization, molecular dynamics and Monte Carlo simulations, previously unthinkable. Here, we introduce the theory and software developments and in the field and overview the applications, the most encouraging results being obtained in the field aqueous chemistry. Requiring state-of-the-art computer resources PES sampling with manybody electronic structure methods in the condensed phase provides highquality benchmarks and will gradually become more available due to fast progress in reduced scaling algorithms and computational technologies.
\end{abstract}

Keywords: electronic structure, potential energy surfaces, wave functions, perturbation theory, random-phase approximation, molecular dynamics, geometry optimization, quantum chemistry

\section{INTRODUCTION}

Electronic structure of the condensed-phase systems has for a long time belonged to the solid state physics domain, whose main interest is band structure of solids. Such calculations were often performed for fixed geometric structures. For modelling the majority of chemically relevant phenomena, such as adsorption, phase transitions, solvation, chemical transformations etc. exploration of the potential-energy surface (PES) is a must [1]. This can be accomplished via Monte-Carlo (MC) sampling, geometry optimization (or structure relaxation) and molecular dynamics (MD). Whereas MC is based on electronic energy calculation, MD requires the energies and forces,

(Dr. V. V. Rybkin) Department of Chemistry, University of Zurich, WinTERThurerstrasse 190, CH-8057 Zurich, Switzerland

E-mail address: vladimir.rybkin@chem.uzh.ch. 
i.e. geometric energy gradients. There exist gradient and non-gradient geometry optimization methods, although the former are much more popular.

A seminal achievement enabling ab initio MD (AIMD) calculations in the condensed-phase was the Car-Parrinello MD (CPMD) [2]. Based on the density functional theory (DFT), a computationally cheaper alternative to many-body electronic structure theories, CPMD employed classical propagation of both nuclear and electronic degrees of freedom and allowed for large-scale simulations at achievable computational cost. Later, due to the progress in computational algorithms and hardware, a more strict BornOppenheimer MD (BOMD) [3], solving the electronic structure problem at every time step, gained popularity. The majority of AIMD simulations currently published are DFT-based BOMD calculations.

In molecular quantum chemistry typically dealing with smaller systems, PES sampling with many-body electronic-structure theory methods is much more common to the extent that only these methods are often regarded as $a b$ initio as contrast to DFT. Indeed, many practical DFT approximations are based on ad hoc assumptions and/or empiricism. Recent developments in computational algorithms, software and hardware have recently allowed for PES sampling with many-body electronic structure methods also in the condensed phase. Such simulations are currently at the cutting edge of molecular simulations and are not numerous. In this minireview, we discuss these computational techniques, introduce software implementations and overview published applications.

\section{THEORY}

2.1. Correlated Wave Function (CWF) Methods. A single Slater determinant Hartree-Fock wave function is defined as non-correlated. Manybody methods introduce electron correlation employing many-determinant expansion of the wave function by applying excitations to the reference Slater determinant [4]. This can be done systematically yielding hierarchies of models of increasing complexity and accuracy. In the limiting case, all possible excited determinants are included, the wave function known as full configuration interaction being exact in the given basis.

2.1.1. Second-order Møller-Plesset perturbation theory (MP2). Second-order Møller-Plesset perturbation theory (MP2) [5] is the simplest ab initio correlated wave function method. It is a variant of many-body perturbation theory with the Fock operator serving as an unperturbed Hamiltonian and applied to the Hartree-Fock reference determinant. Consequently, the zerothorder energy is the sum of orbital energies, the first-order energy is the Hartree-Fock energy, whereas the electron correlation is being computed starting from the second order. Perturbations of higher orders are practically unused. In MP2, the correlation energy $E^{(2)}$ for a closed shell system 
is given by:

$$
E^{(2)}=-\sum_{i \leq j}^{o}\left(2-\delta_{i j}\right) \sum_{a b}^{v} \frac{(i a \mid j b)[2(i a \mid j b)-(i b \mid j a)]}{\epsilon_{a}+\epsilon_{b}-\epsilon_{i}-\epsilon_{j}}
$$

where $i, j \ldots$ run over occupied orbitals, $a, b \ldots$ run over virtual orbitals, $\Delta_{i j}^{a b}=\epsilon_{a}+\epsilon_{b}-\epsilon_{i}-\epsilon_{j}\left(\epsilon_{a}\right.$ and $\epsilon_{i}$ are orbital energies), (ia $\left.\mid j b\right)$ are electron repulsion integrals in the Mulliken notation, and $\delta_{i j}$ is the Kronecker delta function. MP2 includes double excitations and captures most of the dynamic correlation.

In a canonical MP2 energy algorithm the time limiting step is the computation of the $(i a \mid j b)$ integrals obtained from the electron repulsion integrals over atomic orbitals $(\mu \nu \mid \lambda \sigma)$ via four consecutive index integral transformations. The computational effort for the first quarter transformation is $O\left(o n^{4}\right)$, making the MP2 energy calculation a method scaling as $O\left(N^{5}\right)$. The application of the resolution-of-identity (RI-) approximation to MP2 is straightforward [6] and consists in replacing $(i a \mid j b)$ integrals with the approximated $(i a \mid j b)_{R I}$ given in Eq. 2:

$$
(i a \mid j b)_{R I}=\sum_{P} B_{P}^{i a} B_{P}^{j b}
$$

B is a matrix with $o v$ rows and $N_{a}$ columns, given by:

$$
B_{P}^{i a}=\sum_{R}(i a \mid R) L_{P R}^{-1}
$$

where $P, R \ldots$ (total number of them is $N_{a}$ ) are auxiliary basis functions and $\mathbf{L}$ are two-center integrals over the. The computation of $(i a \mid j b)_{R I}$ requires $O\left(o^{2} v^{2} N_{a}\right)$ operations implying that the RI-MP2 method is also scaling $O\left(N^{5}\right)$ with a lower prefactor: the main reason for the speed-up in RI-MP2 lies in the strongly reduced integral computation cost.

2.1.2. Direct Random Phase Approximation (RPA) Correlation Energy Method. The RPA correlation energy is given as the difference between the zero point energy of two harmonic oscillator excitation problems for which the first includes a correlated ground state (RPA) and the second not [7-9]. RPA is similar to MP2 as it involves only double electron excitations. Unlike MP2, RPA can be applied to any KS reference determinant, which is often the case in practical calculations.

Within the direct-RPA (dRPA) approach, which is RPA without exchange contributions [10] $E_{c}^{\mathrm{dRPA}}$ can be expressed in terms of a frequency integral

$$
E_{c}^{\mathrm{dRPA}}=\frac{1}{2} \int_{-\infty}^{+\infty} \frac{d \omega}{2 \pi} \operatorname{Tr}(\ln (\mathbf{1}+\mathbf{Q}(\omega))-\mathbf{Q}(\omega))
$$


with the frequency dependent matrix $\mathbf{Q}(\omega)$ in the RI basis which is determined by

$$
Q_{P Q}(\omega)=2 \sum_{i}^{o} \sum_{a}^{v} B_{P}^{i a} \frac{\varepsilon_{a}-\varepsilon_{i}}{\omega^{2}+\left(\varepsilon_{a}-\varepsilon_{i}\right)^{2}} B_{Q}^{i a} .
$$

For a given $\omega$, the computation of the integrand function in Eq. 4 using Eq. 5 requires $O\left(N^{4}\right)$ operations. The integral of Eq. 4 can be efficiently calculated by numerical quadrature. Thus, the introduction of the resolution of the identity (RI) approximation and the frequency integration techniques for computing $E_{c}^{\mathrm{dRPA}}$ leads to a reduction of the computational cost to $O\left(N^{4} N_{q}\right)$, where $N_{q}$ is the number of quadrature points [11].

2.1.3. Coupled-cluster (CC) methods. In molecular quantum chemistry, coupled cluster methods are considered the most accurate and robust approaches within the single reference methods [4]. They constitute a systematically improvable hierarchy of approximations based on the famous exponential ansatz:

$$
\left|\Psi^{C C}\right\rangle=e^{\hat{T}}\left|\Psi^{H F}\right\rangle
$$

where $\left|\Psi^{H F}\right\rangle$ is the reference Hartree-Fock determinant and $\hat{T}=\sum_{m}^{n} \hat{T}_{m}$ is the cluster operator. $m$ is the order of excitation, whereas $n$ defines the order of the CC approximation, i.e. $\hat{T}=\hat{T}_{1}+\hat{T}_{2}$ yields the CCSD (singles and doubles). Since the computational complexity with the size of the system scales at least as $N^{6}$ for CCSD and steeper for more accurate approximations, the available practical implementations for the condensed phase are restricted to CCSD and CCSD(T) (perturbative triples correction) and the practically achievable system sizes are limited. For an excellent review of periodic CC methods see Ref [12].

2.1.4. Local correlation methods. It is possible to reformulate canonical manybody methods in terms of localized molecular orbitals. Such approach simplifies the calculation since the contribution of the correlation between spatially distant local orbitals to the total correlation energy is very small and can be neglected. Such approaches reduced computational cost and scaling and, in fact, the first available periodic MP2 implementation was the local method of Pisani et al. Existing local methods are based on Gaussian basis functions and are restricted to the system with large band gaps. For an excellent review of the local wave function correlation methods for the condensed-phase systems, see Ref [13].

2.2. Density functional theory. Density functional theory is an alternative approach to the electron correlation problem [14]. Within DFT the ground state energy and properties can be exactly expressed as a functional of electron density, although the functional form is not known. The most widespread DFT realization - Kohn-Sham (KS) DFT [15] - introduces electron correlation via exchange correlation functionals, still being based on 
the single Slater determinant, i.e. at the cost of Hartree-Fock calculation or even cheaper.

It is convenient to classify electronic structure methods using the Perdew's "Jacob's ladder" [16], each rung of it introducing more descriptors and yields models with improved accuracy:

(1) the electronic density,

(2) its gradient,

(3) the kinetic energy density,

(4) the occupied molecular orbitals (MO), usually in form of HartreeFock exchange, and

(5) the unoccupied or virtual MOs.

Generalized-gradient approximation (GGA), the working horse of molecular simulation, belongs to the second rung, while hybrid functionals belong to the fourth rung. At the fifth rung the inclusion of the virtual orbitals allows for taking into account non-local, dynamic, electron correlation, that contribute to the long-range van der Waals (vdW) dispersion interactions. These along with the large self-interaction error are known fallacies of GGA DFT. Exactly these properties are crucial for accurate simulation of electron transfer and aqueous systems. Many of the various functionals on the $5^{\text {th }}$ rung are based on either the random phase approximation (RPA) [9] or second-order Møller-Plesset perturbation theory (MP2) [17], in the form of double hybrids $(\mathrm{DH})$. Indeed, using virtual orbitals means applying excitations to the reference KS determinant exactly as in the many-body wave function theories, which makes a link between many-body methods and DFT.

2.3. Periodic boundary conditions and basis sets. Condensed-phase simulations are normally performed under periodic boundary conditions (PBC), making "condensed-phase" and "periodic" synonymous in this context. A natural choice of one-electron basis under PBC are plane waves (PW). PW are periodic, complete and orthonormal, that leads to efficient integral evaluation, simple energy derivatives expressions (including forces) and systematic convergence to the basis set limit [18-20]. However, they do not reflect the chemical nature of molecular systems. Thus, one requires a large PW basis for accurate calculations. This may turn detrimental for CW methods since the basis size defines the size of the virtual space, which is critical for the computational performance.

Alternatively, one may use local (atom-centered) Gaussian basis functions, a standard choice of molecular quantum chemistry [4]. Designed to reflect atomic shells and describe chemical bonding they provide a compact orbitals expansions, density matrices and smaller virtual spaces. Gaussians are not complete, i.e. it is not trivial to design a quality basis set and converge to the basis limit. In addition, Gaussians depend on atomic positions, that makes expression for the forces more complicated as in the PW basis. It is possible to evaluate integrals over Gaussian basis functions efficiently 
by the Gaussian and plane waves method (GPW) [21], employing auxiliary PW basis set. An alternative type of local basis functions applied for condensed-phase calculations is numerical atom-centered functions [22].

PW calculations often use pseudopotentials for the core levels, due to sharp oscillations of the corresponding orbitals ("hard" orbitals) not properly described by the sparse grid. Thus, GPW method employing auxiliary PW basis is also used with pseudopotentials for the core levels. All-electron calculations are possible within augmented formalism methods [23,24]. Numerical atom-centered functions allow for all-electron calculations by construction [22].

Periodic electronic structure calculations assume sampling of the Brillouin zone by an adequate k-point mesh. Often it suffices to perform the calculation with just $\Gamma$ - point, provided the simulation cell is large enough (a supercell). This is often the case for liquids and interfaces, where periodicity is itself an approximation.

2.4. Geometric energy gradients. As mentioned before, geometrical energy gradients enable geometry optimization and MD simulations. Stress tensors are energy derivatives with respect to periodic cell parameters and needed for varying the periodic cell, i.e. for cell optimization and MD under constant pressure. They can be computed at little additional cost together with the gradients. Since, all CWF methods available for periodic systems are non-variational (at least, in their practical realization) with respect to orbital and many-body expansion coefficients, the analytical evaluation of gradients is rather involved. For the atom-centered Gaussian basis sets, there is another complication due to geometric dependence of of the basis functions. This makes the computation of forces several times more expensive than the computation of energies. Analytical expressions gradients for MP2 [25-28], RPA $[29,30]$ and CC $[31,32]$ are well known from quantum chemistry and are trivially generalized for $k$-points. Due to computational cost, currently only MP2 and RPA gradients are available in the existing software.

Local and fragment correlation methods simplify the calculation at the price of more complicated equations, especially those for derivatives. Therefore, only fragment molecular orbital MP2 forces are available.

\section{Software implementations}

There exist several available program implementations of the many-body correlation methods under PBC. With the PW basis, both MP2 [33,34] and RPA energies [11] and forces [35] are available with k-point sampling in the VASP program $[18,19]$. Related to VASP is the cc4s program featuring CC methods [36] to computed electronic energies [12].

MP2 energies [37,38] and forces [39-41] as well as RPA energies [38,42] with Gaussian basis sets are available (with RI and GPW techniques employed for the integral evaluation, RI-GPW) in the CP2K program. This 
implementation is massively parallel supercomputer architectures and allows for extensive PES sampling (MD and MC) of the systems containing hundreds of atoms in the periodic cell, although it is restricted to the $\Gamma$ point. On thousands of compute cores one can perform energy and force calculations of systems containing several hundreds of atoms.

Another available computational tool is a rapidly developing PySCF suite with MP2, CC [43] and also multi-configurational self-consistent field (MCSCF) methods available with Gaussian basis sets. For MP2, CCSD and $\operatorname{CCSD}(\mathrm{T})$ forces are available for the $\Gamma$-point as as extension of the nonperiodic counterparts of the program [44].

Local correlation energies, MP2 and various CC variants with Gaussian basis sets are available in Crystal/Cryscor [45]. MP2, RPA energies with numerical atom-centered basis functions are implemented in FHI AIMS program [46].

Fragment molecular orbitals MP2 (FMO MP2) energies and gradients in scaled-opposite spin approximation with Gaussian basis sets have also been reported [47].

\section{Applications}

The least expensive way of applying many-body correlation methods to sampling the PES of the condensed-phase systems is to compute single point energies of the structures generated by less expensive methods. Such applications have become accessible and increasingly popular. Reviewing them would thus go beyond the scope of a minireview. Consequently, we will rather concentrate on the applications, where the methods of interest are driving the structural change, i.e. $\mathrm{MD}, \mathrm{MC}$ and geometry optimization based on energies and/forces at the CWF level in the condensed phase.

4.1. Structure of molecular crystals. MP2 captures most of dynamics electron correlation responsible for intramolecular interactions without any empiricism, which makes it a promising method to study molecular crystals. The RI-GPW-MP2 method has been validated for a number of molecular crystals subject to geometry and cell optimization, including carbon dioxide, ammonia, urea, formic acid, benzene, pyromellitic dianhydride, succinic anhydride, and cyclotrimethylene-trinitramine [39]. The obtained results (cell parameters, molecular parameters and cohesive energies) are generally in good agreement with both previously reported calculations and experimental data, although they are sensitive to the basis set size and show notable basis set superposition errors. As a general trend, a good agreement with experimental data is found when the crystals are bound with mixed electrostatic-dispersion interactions (including hydrogen bonds). For crystals of benzene and pyromellitic dianhydride, purely bounded with vdW dispersion interactions, larger deviations are observed, resulting in general in an overestimation of the cohesive energy. 
RI-GPW-MP2 has been applied to the structure of potassium dihydrogen phosphate (KDP) crystals used for higher-order harmonic generation in nonlinear optics [48]. The $2 \times 2 \times 1$ supercell of 64 atoms was studied yielding the geometric H-bonding parameters of the quality compatible to the van der Waals-corrected functionals, for which larger supercells were computed.

The largest and the most challenging molecular crystal optimized with RIGPW-MP2 was a TEMPO radical in the high-spin state, using the $2 \times 1 \times 2$ supercell containing 232 atoms [40]. The results are in an excellent agreement with the experiment and considerably more accurate than empirically dispersion-corrected DFT. The price for this computation is high: ca. 2 hours for one optimization step on 256 compute nodes (CRAY XC30), consisting of 8 CPUs and 1 GPU.

4.2. Properties of water. The most ubiquitous liquid, bulk liquid water, has a reach chemistry and physics manifesting in many anomalous properties. Although those are well characterized experimentally, the atomistic picture of liquid water is far more difficult [49]. There is a long and rich tradition in theoretical simulation of liquid water, the work being mostly based on either empirical potentials or DFT. For a detailed discussion on simulating liquid water the reader is referred to works [50] and [41] and the references therein. Here, we present MD and MC simulations of bulk liquid water based on MP2 and RPA. As shown above, these CWF methods are free of empirical and ad hoc approximations and capture most of the dynamic electron correlation by construction and thus can provide clear and unequivocal benchmarks. The published CWF simulations of liquid water include the following studies:

- RPA- and MP2-based MC at ambient conditions in an NpT ensemble ( $T=295 \mathrm{~K}$ and $p=1$ bar $)$ in a cubic periodic cell containing 64 water molecules within the RI-GPW formalism with a triple-zeta basis sets [51,52]; MC involved 17500 cycles for the MP2 simulation and over 30000 cycles for the RPA simulation.

- MP2-driven MD study in an pre-equilibrated NVE ensemble with the analogues settings; two production runs were carried out for 10 ps [52]

- FMO spin-component-scale MP2-driven MD study in an NVT ensemble (at $300 \mathrm{~K}$ ) in a cubic periodic cell containing 32 water molecules with augmented double-zeta Gaussian basis; the sampling time of the production run was 5 ps [47].

The result of RPA and MP2 simulations are encouraging. Table 1 summarizes the density and structural features of the radial distribution functions (RDF) of liquid water obtained from NpT-MC simulations at different levels of theory $[51,52]$ together with the results obtained by standard GGA and hybrid functionals (PBE, PBE0) with D3 [53] and non-local van der Waals (vdW) corrections $[54,55]$. The reported average values show the slight overestimation when GGA and hybrid functionals with the D3 vdW corrections 


\begin{tabular}{lccccccc}
\hline & Density $[\mathrm{g} / \mathrm{mL}]$ & \multicolumn{2}{c}{$1^{\text {st }}$ Maximum } & \multicolumn{2}{c}{$1^{\text {st }}$ Minimum } & \multicolumn{2}{c}{$2^{\text {nd }}$ Maximum } \\
& $\rho$ & $r, \AA$ & $g_{O O}(r)$ & $r, \AA$ & $g_{O O}(r)$ & $r \AA$ & $g_{O O}(r)$ \\
\hline PBE-D3 & 1.055 & 2.73 & 3.07 & 3.25 & 0.69 & 4.43 & 1.21 \\
PBE0-D3 & 1.053 & 2.74 & 2.88 & 3.29 & 0.79 & 4.32 & 1.21 \\
optB88-vdW & 1.081 & 2.74 & 2.94 & 3.34 & 0.80 & 4.31 & 1.21 \\
MP2 & 1.020 & 2.76 & 3.05 & 3.32 & 0.72 & 4.41 & 1.21 \\
RPA & 0.994 & 2.78 & 2.93 & 3.41 & 0.78 & 4.49 & 1.19 \\
exp. & 1.00 & 2.80 & 2.57 & 3.45 & 0.84 & 4.5 & 1.12 \\
\hline
\end{tabular}

TABLE 1. Average Density and structural data obtained from the NpT-MC simulations $(T=295 \mathrm{~K}$ and $p=1$ bar $)$ The density error is estimated to be in the third digit. The optB88-vdW method represents a functional of the non-local van der Waals type $[54,55]$. The label D3 stands for a dispersion correction according to [53].

are used. For the considered non-local vdW functional the obtained results show a larger density of $\sim 8 \%$, and the structure of the liquid is rather well reproduced but with all features shifted to shorter distances, in agreement with previous NVT simulations [56]. Both the inclusion of the D3 correction and the use of hybrid functionals reduces the height of the first peak of the $g_{O O}(r)$ for PBE. This combined effect of dispersion and exchange has been emphasized recently [57]. GGA functionals, which underestimate the band gap, lead to a too polarizable solvent, which in turn should lead to too strong hydrogen bonds. This is similar to the effect of charge transfer from anions to the solvent that has been observed to be too strong for GGAs as compared to hybrids. [58].

It is worth noticing, that density functionals without dispersion correction result in a large underestimation of the liquid water density, predicting, as a result, ice sinking in water. MP2 and RPA reproduce the densities of liquid water and of ice accurately [59], eliminating this non-physical effect.

Dynamical properties - infrared spectrum [47,52], Raman spectrum [47] and diffusion constant $[47,52]$ - computed from MP2 MD trajectories are in good agreement with the experimental values, the spectrum reproducing both fundamental bands and overtones.

4.3. Bulk hydrated electron. Excess aqueous electron, or the hydrated electron, $\mathrm{e}_{\mathrm{aq}}{ }^{-}$, is a species of pivotal in radiation and plasma chemistry [60]. Due to the short life-time and low concentration its structure is inaccessible to direct experimental observation and had for the long time been elusive, opening wide possibilities for theory. This is hardly less challenging as the reliable description should an be based on the MD simulations under PBC (i.e. in the bulk) based on correlated wave function method, providing accurate description of liquid water and free of delocalization error [61], the characteristic of DFT. MP2 satisfies these requirements and the GPW-RI-MP2 
implementation allows such simulations, although at a high computational price.

In work [62], MD simulations of the bulk hydrated electron in the periodic cell of 47 water molecules at MP2 level with triple-zeta quality basis set are reported for the total of ca. 5 ps using the RI-GPW-MP2 implementation in the CP2K program. One MD step for such a simulation required ca. 20 minutes of wall time on 256 compute nodes (CRAY XC50), consisting of 12 CPUs and 1 GPU. Two trajectories initialized by adding an access electron to neat liquid water were run for 2 ps and found a delocalized nature for $\mathrm{e}_{\mathrm{aq}}{ }^{-}$at $0 \mathrm{ps}$, a 5 -coordinated cavity at $0.5 \mathrm{ps}$ converting to a 4-coordinated cavity at $1 \mathrm{ps}$ as shown in Figure 1 . Another trajectory was started from a preformed four-coordinated tetrahedral cavity and showed its stability since the cavities of different types were observed only as fluctuations.

The computed hydrated electron's gyration radius, $2.02 \AA$ and $2.16 \AA$ for different trajectories, coordination number slightly larger than 4 and the regions of negative spin density are in good agreement with experimental data [63-65]. Importantly, these simulation not based on any empiricism, do not confirm the existence of any stable non-cavity structures actively discussed during the last decade [66-69].

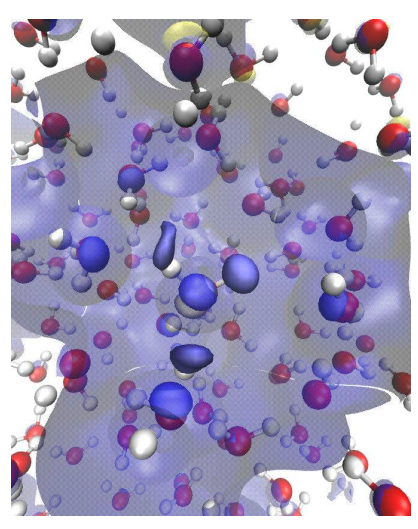

0 ps: initial delocalized structure

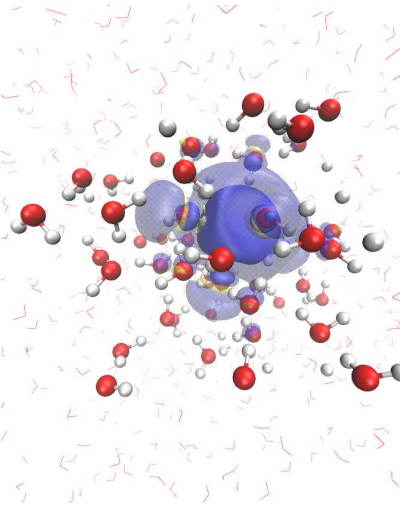

0.5 ps: fivecoordinated four $\mathrm{H}-\mathrm{H}$ bond cavity

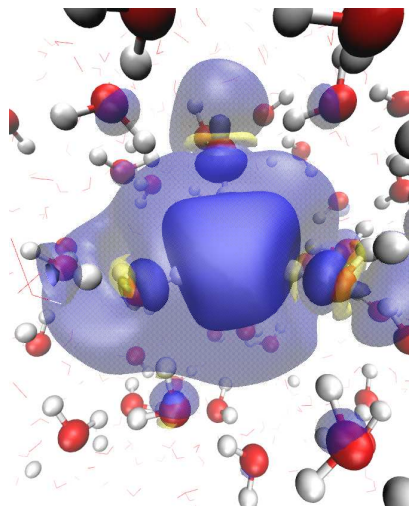

1 ps: four-coordinated three $\mathrm{H}-\mathrm{H}$ bond cavity

FiguRE 1. Dynamic structure of the hydrated electron. Evolution of the hydrated electron's spin density exhibits rapid cavity formation. Blue: positive spin density; yellow: negative spin density. Spin density isovalues: opaque - \pm 0.0015 a.u.; transparent - \pm 0.0001 . The figure is reproduced from reference [62] with the permission of the publisher. 


\section{Conclusions And outlook}

We have presented correlated wave function methods, which have recently become available for simulating matter in the condensed phase, with the focus on sampling PES. These methods can be now applied to liquids, solutions, crystals, and interfaces. The available results of such simulations are encouraging, as they provide a accurate description of systems bound with mixed electrostatic-dispersion interactions (including hydrogen bonds) in a non-empirical fashion. Aqueous systems are a particular important class of such applications. Excellent accuracy is achieved not only in simulations of neat liquid water, but also beyond it, e.g. for aqueous excess electron.

The presented simulations are computationally demanding and can be currently performed only on large supercomputers. However, with the constant increase in computational power, rapid progress in reduced-scaling algorithms and improvement of software the availability of these techniques will be gradually increasing.

\section{ACKNOWLEDGEMENTS}

The author acknowledges the financial support by the Swiss National Science Foundation in the form of Ambizione grant No. PZ00P2_174227. This research was partially supported by NCCR MARVEL funded by the Swiss National Science Foundation. 


\section{BIOGRAPHY}

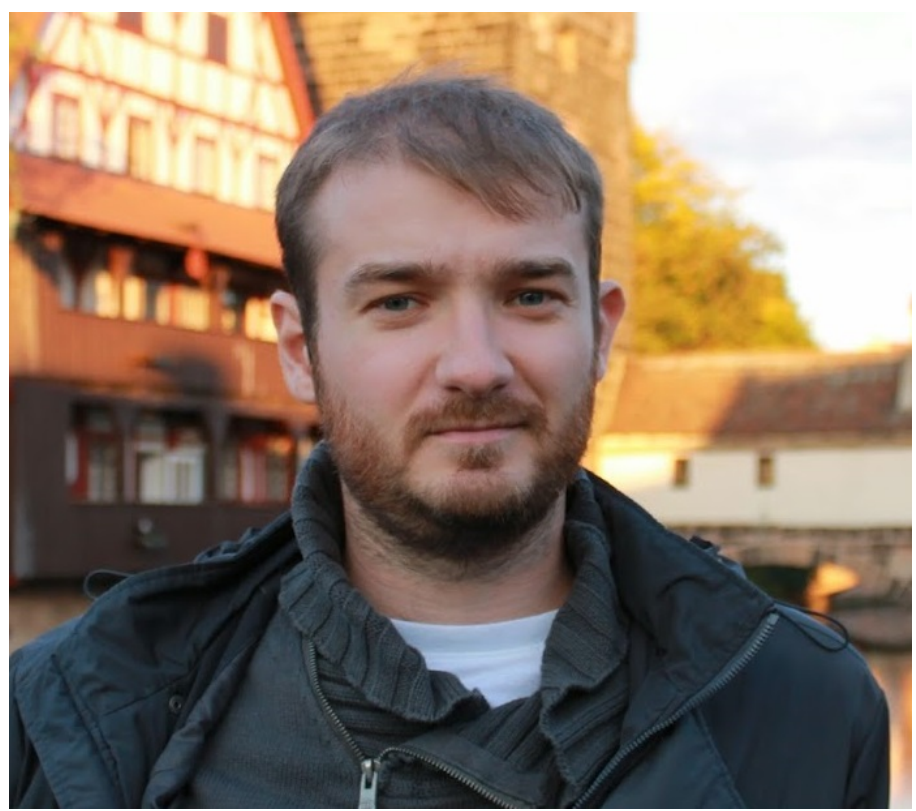

Vladimir V. Rybkin received his M. Sc. degree from Ivanovo State University of Chemistry and Technology, Russia, under supervision of Prof. G. V. Girichev. He has received his PhD from University of Oslo, with Prof. T. Helgaker and was a postdoc at Karlsruhe University of Technology with Prof. W. Klopper, at ETH Zurich with Dr. J. VandeVondele before joining the group of Prof. J. Hutter at the University of Zurich, where he has a position of a senior researcher funded by the Swiss National Science Foundation in the form of Ambizione grant. His research interests include $a b$ initio molecular dynamics, many-body electronic structure theory and embedding theories. Vladimir Rybkin has contributed to Dalton and CP2K quantum chemistry programs. 
7. Graphical TOC entry
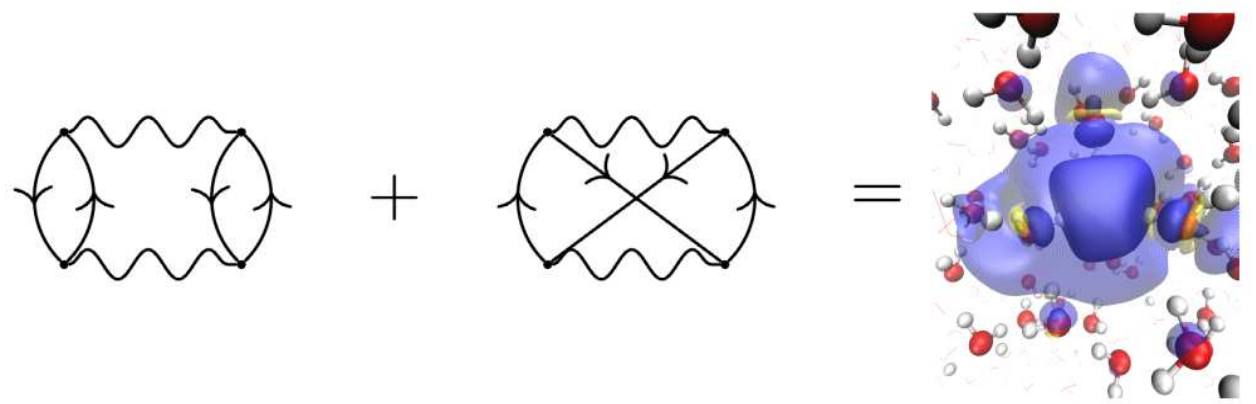


\section{REFERENCES}

[1] D. Wales, Energy Landscapes, Cambridge University Press, Cambridge, 2003

[2] R. Car, M. Parrinello, Phys. Rev. Lett. 1985, 55, 2471-2474

[3] D. Marx, J. Hutter, Ab Initio Molecular Dynamics, Cambridge University Press, Cambridge, 2009

[4] T. Helgaker, P. Jørgensen, J. Olsen, Molecular Electronic Structure Theory, John Wiley \& Sons, Chichester, 2000.

[5] C. Møller, M. S. Plesset, Phys. Rev. 1934, 46, 618-622

[6] M. Feyereisen, G. Fitzgerald, A. Komornicki, Chem. Phys. Lett. 1993, 208, 359-363

[7] F. Furche, J. Chem. Phys. 2008, 129, 114105

[8] G. E. Scuseria, T. M. Henderson, D. C. Sorensen, J. Phys. Chem. 2008, 129, 231101

[9] H. Eshuis, J. E. Bates, F. Furche, Theor. Chem. Acc. 2012, 131, 1084

[10] H. Eshuis, J. Yarkony, F. Furche, J. Chem. Phys. 2010, 132, 234114

[11] M. Kaltak, J. Klimes, G. Kresse, J. Chem. Theory Comput. 2014, 10, 2498-2507

[12] I. Y. Zhang, A. Grüneis, Front. Mater. 2019, 6, 123

[13] D. Usvyat, L. Maschio, M. Schütz, WIRES: Comput. Mol. Sci. 2018, 8, e1357

[14] R. Parr, W. Yang, Density-functional Theory of Atoms and Molecules, Oxford University Press, New-York, Clarendon Press, Oxford, 1989.

[15] W. Kohn, L. J. Sham, Phys. Rev. 1965, 140, A1133A1138

[16] J. P. Perdew, A. Ruzsinszky, and J. Tao, V. N. Staroverov, G. E. Scuseria, G. I. Csonka, J. Chem. Phys. 2005, 123, 062201

[17] S. Grimme, J. Chem. Phys. 2006, 124, 034108

[18] G. Kresse, J. Furthmüller, Comput. Mater. Sci. 1996, 6, 15-50

[19] G. Kresse, J. Furthmüller, Phys. Rev. B 1996, 54, 11169

[20] P. Giannozzi, S. Baroni, N. Bonini, M. Calandra, R. Car, C. Cavazzoni, D. Ceresoli, G.L. Chiarotti, m. Cococcioni, I. Dabo, A. Dal Corso, S. Fabris, G. Fratesi, S. de Gironcoli, R. Gebauer, U. Gerstmann, C. Gougoussis, A. Kokalj, M. Lazzeri, L. Martin-Samos, N. Marzari, F. Mauri, R. Mazzarello, S. Paolini, A. Pasquarello, L. Paulatto, C. Sbraccia, S. Scandolo, G. Sclauzero, A. P. Seitsonen, A. Smogunov, P. Umari, R. M. Wentzcovitch, J. Phys.: Condens. Matter 2009, 21, 395502

[21] G. Lippert, J. Hutter, M. Parrinello, Mol. Phys 1997, 92, 477488

[22] V. Havu, V. Blum, P. Havu, M. Scheffler, J. Comput. Phys. 2009, 228, 8367-8379

[23] P. E. Blöchl, Phys. Rev. B 1994, 50, 17953

[24] G. Lippert, J. Hutter, M. Parrinello, Theor. Chem. Acc. 1999, 2, 124140

[25] J. A. Pople, R. Krishnan, H. B. Schlegel, J. S. Binkley, Int. J. Quantum Chem. 1979, 16, 225241

[26] T. Helgaker, P. Jørgensen, N. Handy, Theor. Chim. Acta 1989, 76, 227245

[27] N. C. Handy, H. F. Schaefer, J. Chem. Phys. 1984, 81, 5031-5033

[28] F. Weigend, Theor. Chem. Acc. 1997, 97, 331-340

[29] J. Rekkedal, S. Coriani, M. F. Iozzi, A. M. Teale, T. B. Pedersen, J. Chem. Phys. 2013, 139, 081101

[30] A. M. Burow, J. E. Bates, F. Furche, H. Eshuis, J. Chem. Theory Comput. 2014, 10, 180-194

[31] L. Adamowicz W. D. Laidig R. J. Bartlett, Int. J. Quant. Chem. 1984, 26 245-254

[32] K. Hald, A. Halkier, P. Jørgensen, S. Coriani, C. Hättig, T. Helgaker, J. Chem. Phys. 2003, 118, 2985-2998

[33] M. Marsman, A. Grüneis, J. Paier, G. Kresse, J. Chem. Phys. 2009, 130184103

[34] T. Schäfer, B. Ramberger, G. Kresse, J. Chem. Phys. 2017, 146104101

[35] B. Ramberger, T. Schäfer, G. Kresse, Phys. Rev. Lett. 2017, 118, 106403

[36] F. Hummel, T. Tsatsoulis, A. Grüneis, J. Chem. Phys. 2017, 146, 124105

[37] M. Del Ben, J. Hutter, J. VandeVondele, J. Chem. Theory Comput. 2012, 8, 41774188 
[38] M. Del Ben, J. Hutter, J. VandeVondele, J. Chem. Theory Comput. 2013, 9, 26542671

[39] M. Del Ben, J. Hutter, J. VandeVondele, J. Chem. Phys. 2015, 143, 102803

[40] V. V. Rybkin, J. VandeVondele, J. Chem. Theory Comput. 2016, 12, 2214-2223

[41] J. Hutter, J. Wilhelm, V. V. Rybkin, M. Del Ben, J. VandeVondele in Handbook of Molecular Modeling: Theory and Modeling (Eds.: W. Andreoni, S. Yip), Springer, 2018, pp. 1-21

[42] J. Wilhelm, P. Seewald, M. Del Ben, J. Hutter, J. Chem. Theory Comput. 2016, 12, 5851-5859

[43] J. McClain, Q. Sun, G. K.-L. Chan, T. C. Berkelbach, J. Chem. Theory Comput. 2017, 13, 1209-1218

[44] Q. Sun, T. C. Berkelbach, N. S. Blunt, G. H. Booth, S. Guo, Z. Li, J. Liu, J. D. McClain, E. R. Sayfutyarova, S. Sharma, S. Wouters, G. K.-L. Chan, WIRES: Comput. Mol. Sci. 2017, 8, e1340

[45] C. Pisani, M. Schütz, S. Casassa, D. Usvyat, L. Maschio, M. Lorenz, A. Erba, Phys. Chem. Chem. Phys. 2012, 14, 7615-28

[46] V. Blum, R. Gehrke, F. Hanke, P. Havu, V. Havu, X. Ren, K. Reuter, M. Scheffler, Comput. Phys. Comm 2009, 180, 2175-2196

[47] S. Y. Willow, M. A. Salim, K. S. Kim, S. Hirata, Sci. Rep. 2015, 5, 14358

[48] R. Menchon, G. Colizzi, C. Johnston, F. Torresi, J. Lasave, S. Koval, J. Kohanoff, R. Migoni, Phys. Rev. B 2018, 98, 104108.

[49] J. R. Errington, P. G. Debenedetti, Pablo G. Nature 2001, 409, 318-321

[50] M. J. Gillian, D. Alfe, A. Michaelides, J. Chem. Phys. 2016, 144, 130901

[51] M. Del Ben, J. Hutter, J. VandeVondele, J. Phys. Chem. Lett. 2013, 4, 3753-3759

[52] M. Del Ben, J. Hutter, J. VandeVondele, J. Chem. Phys. 2015, 143, 054506

[53] S. Grimme, J. Antony, S. Ehrlich, H. Krieg, J. Chem. Phys. 2010, 132, 154104

[54] J. Klimeš, D. Bowler, A. Michaelides, J. Phys.: Condens. Matter 2010 2010, 22, 022201

[55] J. Klimeš, D. Bowler, A. Michaelides, Phys. Rev. B 2011, 83, 1-13

[56] J. Wang, G. Román-Pérez, J. M. Soler, E. Artacho, M.-V. Fernández-Serra, J. Chem. Phys. 2011, 134, 024516

[57] R. A. DiStasio, B. Santra, Z. Li, X. Wu, R. Car, J. Chem. Phys. 2014, 141, 084502

[58] J. VandeVondele, P. Troester, P. Tavan, G. Mathias, J. Chem. Phys. A 2012, 116, 2466-2474

[59] M. Del Ben, J. VandeVondele, B. Slater, J. Phys. Chem. Lett. 2014, 5, 4122-4128

[60] J. M. Herbert, M. P. Coons, Annu. Rev. Phys. Chem. 2017, 68, 447-472

[61] P. Mori-Sanchez, A. J. Cohen, W. Yang, Phys. Rev. Lett. 2007, 100, 146401

[62] J. Wilhelm, J. VandeVondele, V. V. Rybkin, Angew. Chem. Int. Ed. 2019, 58, 38903893

[63] L. D. Jacobson, J. M. Herbert, J. Am. Chem. Soc. 2010, 132, 10000-2

[64] D. M. Bartels, J. Chem. Phys. 2001, 115, 4404-4405

[65] W. Fessenden, N. C. Verma, J. Am. Chem. Soc. 1976, 98, 243-246

[66] R. E. Larsen, W. J. Glover, B. J. Schwartz, Science 2010, 329, 65-69

[67] L. D. Jacobson, J. M. Herbert, Science 2011, 331, 1387-1387

[68] L. Turi, A. Madarasz, Science 2011, 331, 1387-1387

[69] R. E. Larsen, W. J. Glover, B. J. Schwartz, Science 2011, 331, 1387-1387 